\title{
An Investigation on Perspective of Usability and Functionality of Mobile Phones for Elderly
}

\author{
S. T. Raza and F. Sahar
}

\begin{abstract}
Ageing population's increasing ratio is alarming. Fast increase in ageing population means the growing of chronic diseases as well. Diabetes type 2 is a chronic disease which commonly found in elder persons. Technological innovations in health care sector playing an enormous role in improving the healthy life of all humans. Use of latest technologies in perceiving the health services are now common. The focus of this study is to investigate how the features of usability help in designing the mobile phone for elder persons with minimum complexities. The study is based on the literature review with the perspective of usability and functionality of the mobile phone. In this part we do not focus on usability as a whole because usability itself is broad field, but we mainly target the usability attributes which helps in designing the simple mobile phones for elder persons. We will describe the usability attributes which are necessary in the phase of designing new mobile phone for elder persons. The combined study of functionality of mobile phone and its usefulness will be helpful for the designers and manufacturers of mobile phones in understanding the future requirements of the users.
\end{abstract}

Index Terms-Elderly, functionality, mobile phones, usability

\section{INTRODUCTION}

Technology is playing an enormous role in the life of humans. He technology evolution progresses in three stages; tools, machines and automation. This progressive elaboration of technology moves from physical labor to machine controlling and then towards computerization. In third stage humans controlling factor removed with automatic algorithms. The work load and burden of human life is reduced with the use of technology. Mobile Phone is one example of automation which uses for mobile telecommunication. The mobile phone invention makes the lives easier as it finished the distances. Now a day mobile phones are not only using for telecommunication but also providing many other functionalities e.g. SMS, GPRS, MMS etc. According to International Telecommunication Union (ITU), more than $60 \%$ of world's population owns mobile phone [1]. Mobile Phone has broken the restrictions of fixed telephone and allows the people to move anywhere and stay connected all the time. The independence of movement is increased specially in elderly; they can enjoy independent life, travelling without the fear of insecurity due to having mobile phone. According to Kurniawan et. al., Young mobile phone users are increasing very fast but elder users are not growing with expected rate due to unfamiliarity with new mobile phones. This gap of unfamiliarity with mobile phones causes

Manuscript received January 9, 2013; revised April 19, 2013.

The authors were with Blekinge Institute of Technology, SE- 37179 Karlskrona, Sweden (e-mail: farrukhsahar@yahoo.com, tosif@live.com). serious side effects to elder. The use of mobile phones in people of age 75 and above is only $24 \%$. Elder people use mobile phones only for calling to their relatives and sometime for emergency. This gap of unfamiliarity with mobile phones is harmful for both elderly and mobile phone manufacturing companies. The average population of elder people is increasing with smooth percentage. According to the statistics of the United Nations, the world's population with age of 60 years and above was $9.9 \%$ in 2000 , which will increase up to $11 \%$ in 2010 . In 2025 this will be $14.9 \%$ and in 2050 the $21.9 \%$ of the overall population of the world will be above than the age of 60 years. In northern Europe older population is comparatively high with other parts of the world. In 2000 the elder people were $20.4 \%$ which will increase to $22.6 \%$ in 2010 . It will be $26.1 \%$ in 2025 and $29.5 \%$ in 2050 . Sweden is a country where elder population rate is very high comparing with other parts of the world. The statistics shows, elder population was $22.1 \%$ in 2000 which increased to $23.3 \%$ in 2005 , the expected elder population in 2025 is $27.8 \%$ in Sweden [2].

\section{LITERATURE REVIEW}

\section{A. Usability}

Usability means the use of a product or system which allows the persons to accomplish their tasks and goals easily and quickly. It applies on every part, aspect, and feature of the product which offers the users to interact with, such as software, hardware, icons, menus etc [3].

Usability is defined by different standards as "the extent to which a product can be used by specified users to achieve specified goals with effectiveness, efficiency and satisfaction in a specified context of use" [ISO 9241-11:1998]

"The capability of the software product to be understood learned, used and attractive to the user, when used underspecified conditions" [ISO/IEC 9126-1, 2000]

"The ease with which a user can learn to operate, prepares inputs for, and interprets output of a system or component" [IEEE Std. 610.12-1990] Usability is term which refers easy interaction of the user with the system. Usability of a system often assessed as how easy it is in operating and learning and how much is satisfied the users [4].

1) Benefits of Usability: According to Pieratti [3] the benefits of usability are important in terms of product quality, cost, and customer satisfaction. Few benefits of usability are following:

- Usability improves the development process through efficient design

- Usability removes the complex functionalities and design the product as per the user requirement 
- A usable product reduce the cost of the product because it minimizes the extra trainings

- A usable product benefits in three ways; it satisfies the user, increase productivity, and reputations both for product and for the organization that developed it [3].

2) Usability of Mobile Phone: Mobile phones are the devices that accumulated the concept of a system in one device. Mobile phones are not only considered as a device but they own system in themselves. Technical complexities in mobile phones need to solve, so they can be made usable and useful for every type of users [5]. Usability depends on easy interaction with system, a mobile phone with usable interface, simple functionality, and good service quality can facilitate much better users especially elder users. The poor interface design, problems in completing tasks, complex functionality etc. not only decreases the value of mobile phone brand but the attraction of network service provider also declines [5].

3) Design and User Perspective: Mobile phone comes in the category of "interactive systems" [5] from design perspective. According to ISO 13407 an interactive system is: "a combination of hardware and software components that receive input from and communicate output to a human user in order to support his or her performance or a task" [5]. From the user perspective a mobile phone is an "information appliance" [5]. Information appliance means to design something for performing activities [5].

4) Universal Usability and Digital Convergence.: Universal usability means that more than $90 \%$ of users have complete information about the system. The complete information can be achieved through high level of interactive with system [5].

Digital convergence:'digital convergence introduces new kind of usability requirements when complex technologies are embedded to everyday appliances" [5]. The interfaces of mobile phones depend on brands, each brand design interface according to its requirements. Only few interface elements in all brands are common, it is necessary to define standards and requirements for design principles and universal usability which all brands adopt [5].

5) Attributes of Usability: It is important to understand that usability is not considered as a single property which deals the user interactions with the system [5]. Usability has several components and which are associated with following attributes of usability:

Learnability: The system should be simple and easy to operate so the user can start working on it without having huge training. The system allows self learning to the users.

Memorability: The functionality of the system should not be complex so the user can remember it easily. When a user who stops using the system for a short period when return to the system can start working easily without having complete training again.

Efficiency: Efficiency of a system can be judged through the high productivity level and it can be achieved when the system will be efficient to use.

Errors: It is difficult to make a system completely error free but it is necessary to make the system with low error rate, so the chances of errors from user minimized.

Satisfaction: The success of any system depends on the users' satisfaction with it. A system which gives the freedom to users in operating subjectively satisfied the user.

These are the key attributes of any system or product which needs to be fulfilled for achieving the maximum usability of that system or product [6].

All these attributes of usability are necessary for less complex functionality of mobile phone. These attributes makes the mobile phone more usable for elderly. Learnability and Memorability of are important factors in functionality because involving of these two attributes means the functionality of mobile phone is easy is easy to learn. A mobile phone which keeps above mentioned attributes can helps elder persons in better way.

6) Mobile phone System Accessibility: Usability is a sub part of system acceptability which ensures that the system is good and fulfills the requirements and needs of the all stakeholders, such as clients, users and managers. The accessibility of a product is depends on its social and practical acceptability [6]. Mobile phone and network services are inter-related with each other, in some cases user face difficulties from network but due to misunderstanding he/she blames to mobile phone interface. Same case applies on problem from mobile phone interface. These kind of issues can b removed with standardize hierarchy which explain and separates the functionalities of mobile phone interface from network services [5].

\section{B. Mobile phone Interfaces}

Ketola, P. and Röykkee, M., [4] defined three conceptual interfaces for the user interaction with mobile phones; they are user interface, service interface, and external interface. For each interface some important elements and attributes are defined.

1) User Interface.:Several factors are involved in building a mobile phone user interface. The factors includes in user interface are input/output techniques, and design factors with industrial and mechanical perspective. Input tools for mobile phone are keypad and voice. Call management and navigation keys are the tools which mobile phone interface needs. Output to users is given through display and voice, display includes localization, indicator, icons, and language familiarity, voice includes ringing tones, quality speakers, and minimum interruption [5].

2) External Interface: External interface is not the physical part of mobile; it just helps in using the mobile phone. It includes accessories, support elements, software add-ins, and PC connectivity. External interface is important part of mobile phone usability but some time omitted at the time of usability engineering [5].

3) Service Interface: The visibility of operator's services to mobile phone users through user interface is known as service interface. Service interface facilitate the users in using different services such as internet service, voice mailbox, data services, and text messaging services. The important factors of in service interface are utility, availability, and interoperability of services [5].

\section{Design And Functionality of Mobile Phone For ELDERLY}

This chapter elaborates the design requirements of mobile phones for elder persons. This chapter also includes mobile 
phone functionality and ageing effects on different capabilities of elder's body. In the end of this chapter common problems of elder person in using mobile phone and motivational factors are discussed. Mobile phone design is directly linked with human interaction with system. In one level the design of mobile phone allows the users to interact with screen and keypad easily. Whereas in other level complex interactions are includes in the design of interfaces according to the context of use. The simplicity of mobile devices evaluated with their successful usability [6].

\section{A. Mobile Phone Design Requirements for Elderly}

Pattison, M. et al., suggests that developers should understand the needs of elder people while they designing of new technologies so, they can live through independent life and interaction with technology. Rather than alienate them because they do not know how to interact with new graphical user interfaces, use internet and mobile phone [7]. According to [5] "As users grow older and their requirements change, designers should be sensitive to their changing user needs as well designing for the users they might eventually become themselves"[6].

\section{B. Performance Capabilities and Facets of Ageing}

By definitions the elderly are the long living users than the young users that's why elder user considered heterogeneous group. The hearing, vision, cognition, and motor functions are the terms which exist as a function of age in performance capabilities [8].

1) Hearing: Gradually decreasing in listening capabilities is most common factor associated with ageing. Decrease in hearing results in many impairments and this decreasing is can be the result of many factors such as genetic influences, work, diet, and most obviously by the age when it turns to above 50( sufficient loss of hearing often occurs at or after this age). Ageing also has effects on the ability of inferring the information from complex hearings. The ability to distinguishing the frequency also get worse gradually in the age of 25 to 55 but after that special devices are required especially in case of higher frequencies [9].

2) Vision: Holzinger, A. et al., describes that visual impairment took place in many forms; it starts from a minor loss to complete blindness. One cause of reducing visual capacity is macular degeneration with increasing the age. Macular degeneration reduces the eyesight which results in partial visual impairment. But this is not an obstacle in the existence of modern technology and can be covered with the help of good interface design [9].Effects on eyesight in older adults can be seen in different ways, some of them are mentioned below:

\section{- Visual Acuity}

It normally starts decreasing after the age of 50 years. The ability to differentiate between the objects decreased and in this case older adult need three times more light for concentration than the average young man [9].

\section{- Contrast Sensitivity}

The decreasing of contrast sensitivity results in reducing the ability to differentiate between the dark and light. It starts from 20 years of age to 80 years but the normally declining of contrast sensitivity starts between the ages of 40 to 50 years [9].

\section{- $\quad$ Focus on Objects}

The ability to focusing on objects starts decreasing from any age between 8 to 50 years normally. In this case person suffers from declining the light accommodation cannot focus on the near and far objects [9].

\section{- $\quad$ Problems with glare}

The increasing in the effects of glare results from the "scattering of light in the eye due to increasing lens opacity. [9]" For elder people three times more light (compared to visual acuity) required to increase the probability of glare needs in design solutions [9].

3) Motor Function: The part of the nervous system that control and regulates the movements of muscle in the body is known as motor function. The strength and power of muscles reduces with the passage of time in functionality and working. When hand motor function loses its strength, it weakens the grip power of the hand. In few cases weakness of hand motor function starts from the age of 30 years but normally the elder people of 65 or above face reduction in motor function. The grip strength of elder people normally reduces up to $25 \%$ in their hands [9].

4) Cognitive Complexity: According to [4] "Kieras and Polson (1985) proposed a theoretical model (Cognitive Complexity Theory (CCT)) specifically dealing with the cognitive complexity of the interaction between user and device by describing the user's goals on the one hand and the reaction of the computer system on the other by means of production rules"[4]. The procedural knowledge and technological applications are indispensable for each other. The usability of the systems helps in acquiring the more knowledge procedures to interact and operate the application efficiently. Ziefle\& Bay's experiment shows that navigations performance of elderly users is lower than the younger users. However, in mobile applications with low complexity the performance of elder users matched with younger [9].

\section{Elderly as Users of Mobile Phone}

It is true that mobile phones are designed with keeping in mind the needs and fashions of young users. But the mobile phone is a technology which never only facilitates a focused group; its benefits are equal for all users. The elder users are ready to increase their sense of freedom and enjoy with quality of life using mobile phones. Furthermore a different utilization of mobile phone for elder people is as a health monitoring tool. In one way mobile phones helps elderly in creating the sense of security and freedom but on other way complex design and functionalities isolate them with society [8].

1) Elder User's Problem with Mobile phone: The elder users are facing several problems with current mobile phones such as too many and complex functions, little buttons, small displays, and difficult keypads. Visual impairments in elder users weaken the capacity to focus on small screens. Cognitive complexities slower down the response time of elderly because of the decline in long term memory with age [8].

2) Limited Knowledge: Another major problem with elder people is the limited knowledge about interfaces. Interfaces are the necessary component of mobile phones. In new models of mobile phones due to advancement and new features other than call, the interfaces becomes complex. The 
young users can understand these interfaces with little effort due to their prior knowledge about the design of mobile phones. Furthermore the switching from one brand to another also troubles in learning new operating styles although there is little change but for elder people it is more than enough [6].

3) Common problems: Following are some common problems which elder person faces using mobile phone [10]: - Rubbery buttons with small size are difficult in pushing - Unnecessary menu options which confuse the elder persons -Complex functions which hard to understand and difficult to remember

- Small display creates vision problems.

\section{Motivational Factors for Elder Persons}

Generally it is acknowledged that adoption of unknown and new technologies in elderly is less than younger. This is one of the reasons they feel uncomfortable. Motivation is defined as psychological construct, motivational issues includes computer literacy, attitude, acceptability etc. Motivation is an essential element for learning [8]. According to Holzinger, A. et al., some researcher says that we can motivate the elder people toward mobile phones after realizing them the benefits of use [8]. The motivational factors are social Influence, Safety and Self Actualization, Enjoyment and support.

\section{CONCLUSION}

The main objective of our study was is to investigate how the features of usability help in designing the mobile phone for elder persons with minimum complexities. To explore all these issues we studied the previous research works in this field and identified the problems. The use of mobile phone in current elder person is not so good. Many issues are involved in this not using. One major factor is complex functionality of latest mobile phones. Another factor is if less technological awareness in elder persons. Physical effects of diabetes on elder persons are also hurdles in carrying of mobile phones. Weakness in motor function, cognitive impairment, and hearing problems in elder persons are also the causes of avoiding mobile phones.

To conduct this research, we adopted the stepwise approach throughout our study. First of all we studied the literature and understand the effects of diabetes on the patients. Moreover we studied the problems of diabetes type 2 elder persons facing in using of mobile phones. Then we studied the standards of mobile phone usability. In literature study we briefly described the attributes of usability and some important attributes which could be helpful in designing mobile phones with less complexity. If these attributes applies in initial phase of designing of mobile phones for elder persons, they could provide successful end product which can be acceptable by the elder persons. We have also studied the factors that effects on bodies of elder persons either they are due to ageing or due to diabetes.

\section{REFERENCES}

[1] J. Nielsen, Usability Engineering, Cambridge, Academic Press, 1993.

[2] United Nation. [Online]. Available: http://www.un.org.

[3] K. M. Qureshi and M. Irfan, "Usability evaluation of e-learning applications, A case study of it's learning from a student's perspective," Master Thesis, Blekinge Institute of Technology 2009.

[4] D. D. Pieratti, Usability and Technical Documentation, Adding quality and value to software application. The Document Company XEROX.

[5] S. Kurniawan, Y. Nugroho, and M. Mahmud, "A study of the use of mobile phones by older persons," in Proc. CHI '06 Extended Abstracts on Human Factors in Computing Systems, pp. 989-994, April 22-27, 2006, Montreal, Quebec, Canada. ACM 1-59593-298-4/06/0004.

[6] M. Pattison and A. Stedmon, "Inclusive design and human factors: Designing mobile phones for older users," PsychNology Journal, vol. 4, no. 3, pp. 267-284, 2006

[7] P. Ketola and M. Röykkee, The Three Facets of Usability in Mobile Handsets, in Proceeding of CHI 2001, Workshop, Mobile Communications: Understanding Users, Adoption \& Design Sunday and Monday, Nokia, Visiokatu 1, FIN-33720 Teampere, Finland.

[8] A. Holzinger, G. Searle, and A. Nischelwitzer, "On Some Aspects of Improving Mobile Applications for the Elderly," in Proceedings of the 4th International Conference on Universal Access in Human Computer Interaction: Coping with Diversity, pp. 923-932, 2007.

[9] M. Ziefle and S. Bay, "How older adults meet complexity: Aging effects on the usability of different mobile phones," Behaviour \& Information Technology, vol. 24, no. 5, pp. 375-389, 2005.

[10] S. Mathiesen, "If I want i can always turn it off," A Study on the Social Impacts of Mobile Phones, 2008.

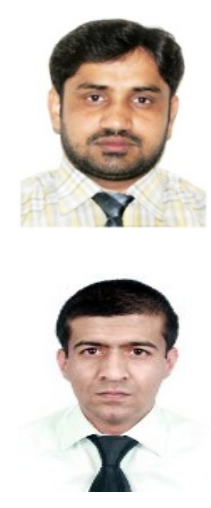

Syed TosifRaza was with Blekinge Institute of Technology, SE- 37179 Karlskrona, Sweden. He is now with the World Health Organization, Regional Office for Europe Copenhagen.

Farrukh Sahar was with Blekinge Institute of Technology, SE- 37179 Karlskrona, Sweden. 